\title{
Arabidopsis lox3 lox4 double mutants are male sterile and defective in global proliferative arrest
}

\author{
Daniela Caldelari · Gaoge Wang • Edward E. Farmer • \\ Xinnian Dong
}

Received: 15 June 2010/Accepted: 30 September 2010/Published online: 3 November 2010

(C) Springer Science+Business Media B.V. 2010

\begin{abstract}
Fertility and flower development are both controlled in part by jasmonates, fatty acid-derived mediators produced via the activity of 13-lipoxygenases (13-LOXs). The Arabidopsis thaliana Columbia-O reference genome is predicted to encode four of these enzymes and it is already known that one of these, LOX2, is dispensable for fertility. In this study, the roles of the other three 13-LOXs (LOX3, LOX4 and LOX6) were investigated in single and double mutants. Four independent lox3 lox4 double mutants assembled with different mutated lox 3 and lox4 alleles had fully penetrant floral phenotypes, displaying abnormal anther maturation and defective dehiscence. The plants were no longer self-fertile and pollen was not viable. Fertility in the double mutant was restored genetically by complementation with either the $L O X 3$ or the LOX4 cDNAs and biochemically with exogenous jasmonic acid. Furthermore, deficiency in $L O X 3$ and LOX4 causes developmental dysfunctions, compared to
\end{abstract}

Electronic supplementary material The online version of this article (doi:10.1007/s11103-010-9701-9) contains supplementary material, which is available to authorized users.

D. Caldelari - G. Wang $\cdot$ X. Dong

Department of Biology, Duke University, Durham,

North Carolina, USA

D. Caldelari $(\bowtie) \cdot$ E. E. Farmer

Plant Molecular Biology, University of Lausanne,

Biophore, CH-1015, Lausanne, Switzerland

e-mail: daniela.caldelari@unil.ch

E. E. Farmer

e-mail: edward.farmer@unil.ch

Present Address:

G. Wang

College of Marine Life Sciences, Ocean University of China,

Qingdao, People's Republic of China wild type; lox3 lox4 double mutants are taller and develop more inflorescence shoots and flowers. Further analysis revealed that developmental arrest in the lox 3 lox4 inflorescence occurs with the production of an abnormal carpelloid flower. This distinguishes lox3 lox4 mutants from the wild type where developmentally typical flower buds are the terminal inflorescence structures observed in both the laboratory and in nature. Our studies of lox3 lox4 as well as other jasmonic acid biosynthesis and perception mutants show that this plant hormone is not only required for male fertility but also involved in global proliferative arrest.

Keywords Lipoxygenase - Jasmonate Male sterility · Carpelloid terminal flower

$\begin{array}{ll}\text { Abbreviations } & \\ \text { LOX } & \text { Lipoxygenase } \\ \text { AOS } & \text { Allene oxide synthase } \\ \text { DAD1 } & \text { Defective in anther dehiscence1 } \\ \text { JA } & \text { Jasmonic acid } \\ \text { LNA } & \alpha \text {-linolenic acid } \\ \text { CaMV 35S promoter } & \text { Cauliflower mosaic virus promoter } \\ \text { WT } & \text { Wild type } \\ \text { FA } & \text { Fatty acid } \\ \text { GPA } & \text { Global proliferative arrest }\end{array}$

\section{Introduction}

Lipoxygenases (LOXs) are found in most eukaryote lineages where they typically catalyze the oxygenation of fatty acids (FAs) containing $1(Z), 4(Z)$ pentadienyl motifs (Schneider et al. 2007). In plants oxygenation can occur on 
carbon number 13 of the FA (reactions catalyzed by the 13LOX family), or in 9-LOX on carbon number 9 (Andreou and Feussner 2009). Through these first canonical LOX reactions hydroperoxy-fatty acids are produced to serve as the precursors of diverse oxygenated fatty acids (oxylipins) including jasmonates in plants. Biologically active jasmonates such as jasmonoyl-L-isoleucine (JA-Ile) are derived through the activity of 13-LOXs and have been shown to be involved in many aspects of defense and development in many plants (Avanci et al. 2010; Browse 2009; Fonseca et al. 2009; Wasternack 2007). Concerning reproduction, the biosynthesis of jasmonates in Arabidopsis during anther development is required to ensure male fertility through controlling stamen filament elongation, pollen development and anther dehiscence. In fact, jasmonate and coronatine (a JA-Ile mimic) perception mutants such as coronatine insensitive1-1 (coil-1; Feys et al. 1994) and jasmonate biosynthetic mutants such as the enzymes allene oxide synthase (aos (dde2); Park et al. 2002; von Malek et al. 2002), 12-oxophytodienoic acid reductase3 (opr3 (dde 1); Stintzi and Browse 2000; Sanders et al. 2000) and the lipase mutant defective in anther dehiscencel (dad1; Ishiguro et al. 2001) are male sterile. Interestingly, the $D A D 1$ gene is itself a target of AGAMOUS (AG), a homeotic protein involved in stamen and carpel development that also plays a secondary post-homeotic role at later stages of flower development involving jasmonate signaling (Ito et al. 2007).

Regarding tissue growth control, it is notable that, in contrast to promoting anther elongation, jasmonates repress petal growth in Arabidopsis (Brioudes et al. 2009). Jasmonates also appear to impact post-fertilization growth events in Arabidopsis since seeds of the aos mutant are larger than those of wild type (WT; Farmer and Dubugnon 2009). The JA-Ile synthesis mutant Osjarl in rice has a strong floral phenotype: the plant is male sterile and, if fertilized with WT pollen, the glumes can no longer cover the developing seed, thus exposing it to the environment (Riemann et al. 2008). All these reported effects concern the impacts of jasmonates on pre- and post-fertilization flower development and seed weight in plants with bisexual flowers. However, in maize, a monoecious plant, jasmonate synthesis was found to be essential for male inflorescence development. Mutations in a $L O X$ gene involved in jasmonate biosynthesis prevented male inflorescence development and resulted in a 'default pathway' to female inflorescence development in the place of male tassels (Acosta et al. 2009). Finally, it is notable that female fertility in Arabidopsis is not affected as strongly as male fertility whereas the opposite is true for tomato $(\mathrm{Li}$ et al. 2001). Together, these findings all underscore the fact that the effects of jasmonate in reproductive development in plants are complex and likely to be somewhat species- dependent. In particular, the recent work on rice and maize raises the possibility that other jasmonate-dependent phenotypes exist in dicotyledons like Arabidopsis, particularly at the level of inflorescence formation and flower development.

The Arabidopsis Columbia-O (Col-O) accession encodes four predicted 13-LOXs: LOX2, LOX3, LOX4 and LOX6 (Bannenberg et al. 2009) and little is known about their specific contributions to jasmonate synthesis. However, recent work has shown that one of these, LOX2, is not required for fertility. Instead, a major role of LOX2 in leaves appears to be the synthesis of jasmonate precursors for incorporation into secondary metabolites known as arabidopsides (Glauser et al. 2009; Seltmann et al. 2010). The roles of the other 13-LOXs in Arabidopsis remain unknown. In this report, we attribute new biological roles to LOX3 and LOX4 demonstrating that these LOX isoforms are necessary for Arabidopsis male fertility. Moreover, we find that these LOXs are required for WT inflorescence development, and indirectly for inflorescence proliferative arrest.

\section{Materials and methods}

\section{Plant materials}

Arabidopsis plants were grown at $22^{\circ} \mathrm{C}$, under a $12 \mathrm{~h}$ light/ day photoperiod. T-DNA insertion mutants were obtained from the Arabidopsis Biological Resource Center (ABRC), and genotyped by allele-specific PCR. At least two different alleles were isolated for each T-DNA insertion mutant, for LOX3 (At1g17420), allele lox3A corresponds to line SALK_119404, allele lox3B corresponds to line SALK_147830, allele lox3D is line SALK_062064, for LOX4 At1g72520, lox4A is SALK_071732, lox4B is SALK_017873, for LOX6 At1g67560, lox6A is SALK_138907 and lox6B is SALK_083650. The silenced LOX2 At3g45140 line and the cognate control line are available as line CS3748 and CS3749, respectively. The dadl mutant was from Ishiguro et al. (2001), and the aos mutant was from Park et al. (2002). The terminal inflorescences of wild Arabidopsis plants (96 individuals) growing on the University of Lausanne campus were observed on May 1, 2010. Only plants that had completed flowering were scored.

Cloning LOX3 and LOX4 cDNAs and plant transformation

$L O X 3$ and LOX4 cDNA clones in the Gateway vector pENTR223 (G21585 and G11274, respectively), were ordered from ABRC. The clones were sequence-verified 
prior to use. Then via an LR Clonase ${ }^{\mathrm{TM}}$ reaction (Invitrogen), each cDNA was introduced into the pMDC32 destination vector (Curtis and Grossniklaus 2003), downstream of the cauliflower mosaic virus (CaMV) $35 \mathrm{~S}$ promoter (Odell et al. 1985). LOX3/pMDC32 or LOX4/pMDC32 were introduced into competent Agrobacterium tumefaciens (strain GV3101::pMP90) using a freeze thaw method (Weigel and Glazebrook 2002). Positive colonies were restreaked onto fresh YEB medium containing Rifampicin $(25 \mathrm{mg} / \mathrm{ml})$, Kanamycin $(50 \mathrm{mg} / \mathrm{ml})$, and Gentamycin $(25 \mathrm{mg} / \mathrm{ml})$ and checked by PCR for the presence of the LOX3 or LOX4 cDNAs. Double mutants lox3 lox4 plants were transformed according to the standard floral dipping protocol. Transformed seedlings were identified approximately 10 days later, by their growth on $1 / 2$ Murahige and Skoog (MS) medium containing $15 \mathrm{mg} / \mathrm{ml}$ of hygromycin.

Recovery of fertility experiments: JA and LNA treatment and pollen viability tests

Flowers of sterile dadl, aos and double lox3 lox4 mutants were dipped in an Eppendorf tube containing either water (control) or $1 \mathrm{mM} \mathrm{JA}$ (jasmonic acid; Sigma-Aldrich) or $0.1 \%$ (v/v) LNA ( $\alpha$-linolenic acid; Sigma-Aldrich), both dissolved in $0.05 \%$ aqueous Tween 20 , for 5 consecutives days.

The pollen viability of WT Col-O plants, single mutants lox3, lox4, double mutants lox3 lox4 and aos mutants was evaluated using a double staining procedure with fluorescein diacetate (FDA) and propidium iodide (PI) as described by Mandaokar and Browse (2009). Pollen grains were examined under a fluorescence microscope (Leica MZ16FA, GFP3 filter for FDA: excitation $470 / 40 \mathrm{~nm}$, emission 525/50 nm, and DsRED filter for PI: excitation $545 / 12 \mathrm{~nm}$, emission at $620 / 60 \mathrm{~nm}$ ). Viable pollen grains fluoresce bright green, while the non-viable pollen grains fluoresce red. In total, 675 WT (Col-0), 872 lox3, 1075 lox4, more than 700 for either lox3 lox4, or aos pollen grains were scored and the percentage of viability calculated.

Substrate binding pocket virtual analysis

The three-dimensional structures of the soybean LOX1 and LOX3 (pdb entries 1YGE, Minor et al. 1996; and 1IK3, Skrzypczak-Jankun et al. 2001, respectively) were superposed using Swiss-PdbViewer (Guex and Peitsch 1997). The primary sequences of the six distinct Arabidopsis LOXs were directly imported into the workspace from Uniprot (Boutet et al. 2007) (accession ids: Q06327, P38418, Q9SMW1, Q9FNX8, Q9LUW0, Q9CAG3; LOX1-6, respectively) and aligned onto the structures using the Align with MUSCLE option of the Fit menu. The residues within 5.0 Angstroms of the 13(S)-hydroperoxy$9(Z), 11(E)$-octadecadienic acid binding site present in the soybean LOX3 were selected and defined as substrate binding pocket (Suppl. Fig. 1), and the corresponding residues of the Arabidopsis LOXs identified from the multiple sequence alignment. Percent identity between the aligned LOXs for the whole sequence and the active site (Fig. 1) have been obtained directly from the Swiss-PDB Viewer alignment window.

\section{Results}

Male sterility phenotypes in lox 3 lox 4 double mutants

LOX3, LOX4 and LOX6 are components of the same phylogenetic clade in Arabidopsis, while LOX2 is disparate (Bannenberg et al. 2009). LOX3 and LOX4 are highly similar, sharing $85 \%$ overall amino acid identity (Fig. 1a and Suppl. Fig. 1). The amino acid sequences of the substrate binding pocket of LOX3 and LOX4 are even more conserved (97\%, Fig. 1b) with only one synonymous amino acid change of a leucine in LOX3 to a valine in LOX4. The strong similarities of LOX3 and LOX4 prompted us to make double mutants for these proteins. We first identified T-DNA insertion mutants for $L O X 3$ (lox $3 A$, lox $3 B$ and lox $3 D$ ), for LOX4 (lox4A, lox4B), for LOX6 (lox6A, lox6B) and the cosuppression line of LOX2 (lox2.S12; Bell et al. 1995). All 13LOX single mutants were fertile (Fig. 2a and Suppl. Fig. 3). We then produced four independent lox3 lox4 double mutants using different insertion mutant lines: lox $3 B$ lox $4 A$,

\section{(A) Full length amino acids sequence identity}

\begin{tabular}{|l|l|l|l|l|l|l|}
\hline & LOX3 & LOX4 & LOX6 & LOX2 & LOX5 & LOX1 \\
\hline LOX3 & $100 \%$ & & & & & \\
\hline LOX4 & $85 \%$ & $100 \%$ & & & & \\
\hline LOX6 & $53 \%$ & $53 \%$ & $100 \%$ & & & \\
\hline LOX2 & $45 \%$ & $45 \%$ & $49 \%$ & $100 \%$ & & \\
\hline LOX5 & $45 \%$ & $45 \%$ & $42 \%$ & $42 \%$ & $100 \%$ & \\
\hline LOX 1 & $45 \%$ & $45 \%$ & $45 \%$ & $45 \%$ & $64 \%$ & $100 \%$ \\
\hline
\end{tabular}

(B) Sequence identity in the substrate binding pocket

\begin{tabular}{|l|l|l|l|l|l|l|}
\hline & LOX3 & LOX 4 & LOX6 & LOX2 & LOX5 & LOX1 \\
\hline LOX3 & $100 \%$ & & & & & \\
\hline LOX4 & $97 \%$ & $100 \%$ & & & & \\
\hline LOX6 & $76 \%$ & $76 \%$ & $100 \%$ & & & \\
\hline LOX2 & $69 \%$ & $69 \%$ & $79 \%$ & $100 \%$ & & \\
\hline LOX5 & $66 \%$ & $62 \%$ & $62 \%$ & $62 \%$ & $100 \%$ & \\
\hline LOX1 & $66 \%$ & $62 \%$ & $62 \%$ & $62 \%$ & $97 \%$ & $100 \%$ \\
\hline
\end{tabular}

Fig. 1 Amino acid sequence identity of Arabidopsis LOX in percentage. a Full length sequence identity. b Sequence identity of the 29 residues composing the substrate binding pocket 
Fig. 2 a Anther phenotype of WT and $L O X$ mutants. b \% of pollen viability. WT (Col-O), lox3, lox4, lox3 lox4, and aos
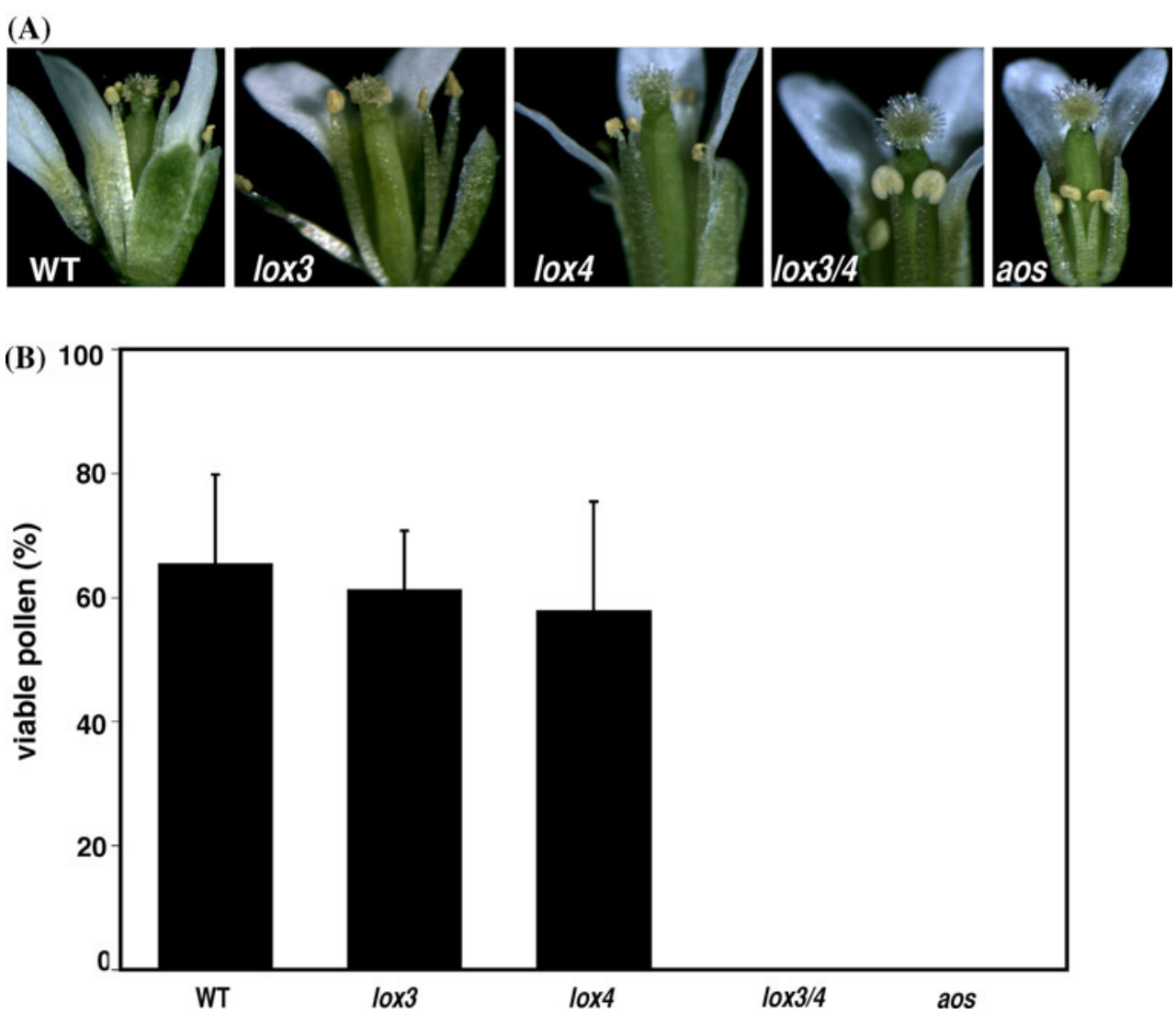

$\operatorname{lox} 3 D \operatorname{lox} 4 B$, lox $4 A$ lox $3 D$, and $\operatorname{lox} 4 B \operatorname{lox} 3 B$. All these mutants were male sterile (Fig. 2a). We also produced lox 3 lox6 and lox4 lox6 double mutants, but these plants were found to be fully fertile (Suppl. Fig. 4). Importantly, all lox3 lox4 double mutants showed the same flower phenotype: shorter anther filaments, indehiscent anthers, and longer stigma papillae than those in WT flowers. We then compared the floral morphology of lox3 lox4 double mutants to that of aos. As shown in Fig. 2a we observed that both mutants show a similar flower phenotype. Moreover, the pollen contained in the indehiscent anthers of lox3 lox4 double mutants and of aos mutants was not viable, while pollen viability in the WT $(65 \%)$ was similar to that in lox3 (61\%) and lox4 (58\%) single mutants (Fig. 2b). Efforts to self-fertilize all lox3 lox4 double mutants with pollen extracted from their own anthers failed. These results indicate clearly that $L O X 3$ and $L O X 4$ are both required for anther and pollen development.

Restoration of fertility to the lox3 lox4 double mutant

In order to confirm the hypothesis that lox3 lox4 double mutants were sterile because they are unable to produce JA indispensable for anther maturation, we applied exogenous JA to the sterile flowers to restore fertility. The flowers were treated either with water, or with $0.1 \%$ (v/v) $\alpha$-linolenic acid (LNA) or with $1 \mathrm{mM}$ JA. LNA was reported to rescue fertility in the lipase mutant $d a d l$, but it did not do so in aos nor in lox3 lox4. In contrast, JA restored fertility in all mutants tested (Fig. 3). Therefore, JA production in the flowers of lox 3 lox 4 double mutants is impaired, resulting in male sterility. Furthermore, the presence of either LOX3 or LOX4 is necessary and sufficient to confer fertility to Arabidopsis, as all the single lox 3 and lox4 mutants are fertile. This is supported by the fact that $\mathrm{T} 1$ plants of lox3 lox4 double mutants expressing the cDNAs of either LOX3 or LOX4 (driven by the strong CaMV 35S promoter) are male fertile (Fig. 4).

\section{Inflorescence morphology}

The JA synthesis mutant aos and all lox 3 lox 4 double mutants were compared and found to share many morphological characteristics. All of these mutants were significantly taller than the WT or the single $L O X$ mutant plants. Under our growth conditions nine-week old WT or single 13-LOX mutant plants measured approximately $35 \mathrm{~cm}$ in height while lox3 lox4 double mutants or aos plants measured more than $50 \mathrm{~cm}$ (Fig. 5a). Moreover, aos or lox3 lox4 double mutant plants developed more floral shoots (on average about 30), compared to WT and single mutants (average around 20). Male-sterile mutants also produced more than 65 flowers compared to 45 flowers for the single mutants or WT 


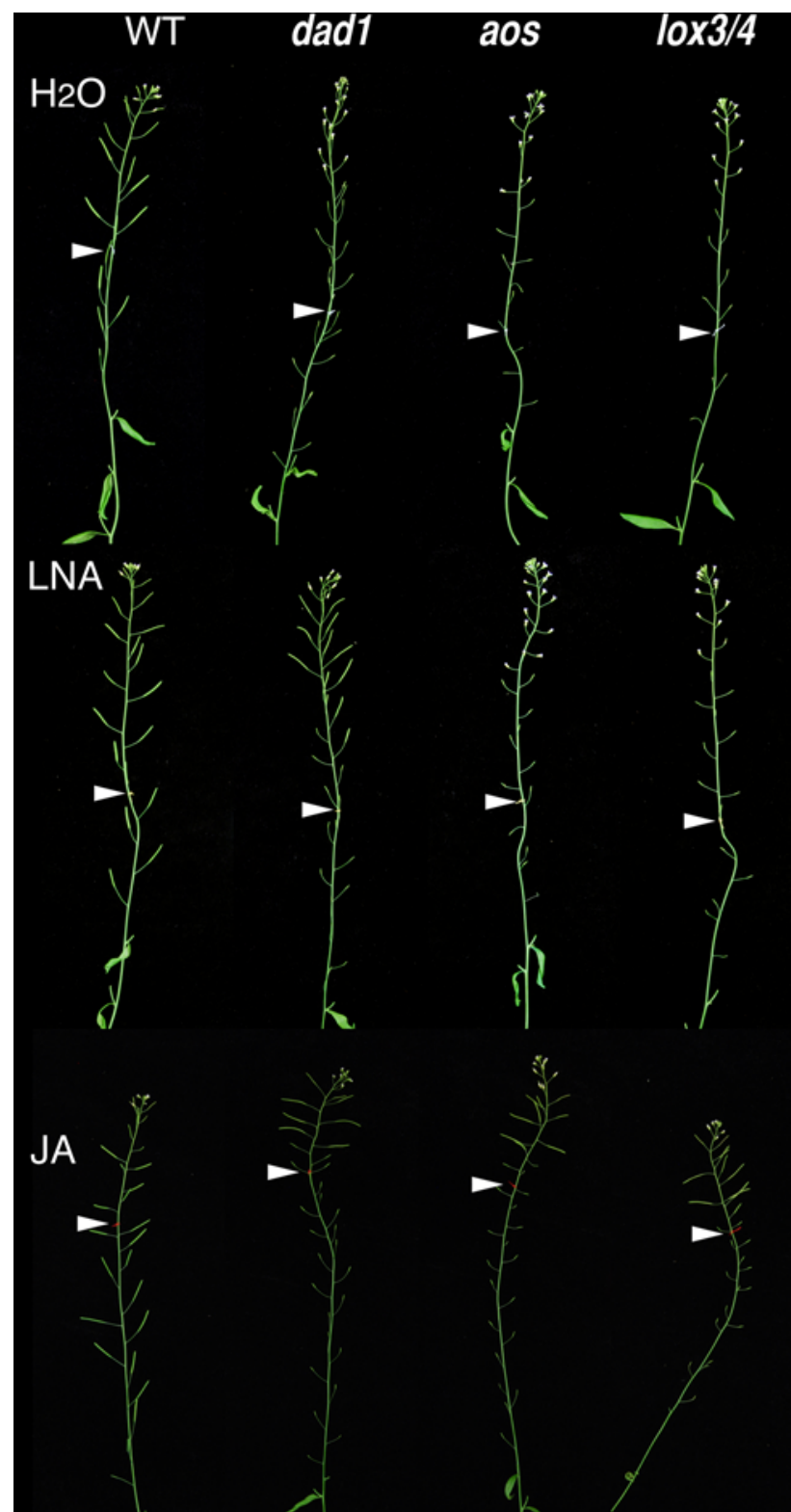

Fig. 3 Restoration of fertility experiments. Arrow heads point to the location where the different treatments were initiated. $\mathrm{H}_{2} \mathrm{O}$ : water, LNA: $0.1 \%(\mathrm{v} / \mathrm{v}) \alpha$-linolenic acid, JA: $1 \mathrm{mM}$ jasmonic acid. The plants used in the experiments were WT (Col-0), dad1, aos, and lox3 lox4

(Fig. 5c). This was due to the fact that aos and lox3 lox4 double mutant continued flowering 1-2 weeks longer than the WT and the single mutants. Consequently, lox3 lox4 and aos plants have a longer life span than the WT under our growth conditions (data not shown).

Floral morphology: the terminal flower of the lox 3 lox 4 inflorescence has carpelloid and staminode structures

In WT plants that have completed flowering and have filled their siliques with seeds, we observed that inflorescence meristematic activity terminated in the production of floral buds (Fig. 6a). In contrast, in the lox3 lox4 double mutant inflorescences, proliferation arrest produced a fully developed abnormal structured flower (Fig. 6e-f). This flower completely lacked petals and sepals which were transformed into open carpelloid structures with exposed ovules and bearing stigmatic papillae. Moreover, the number of stamens was irregular and reduced compared to the WT. Staminode (stamen-like) structures that were larger than WT anthers and of an irregular shape were often present (Fig. 6d-g). We then found the same to be true of $d a d l$, aos, opr3, coil-1 (data not shown), and in WT (Col-O) plants rendered sterile by the surgical removal of fruits (Fig. 6b-d).

\section{Discussion}

$L O X 3$ and $L O X 4$ contribute to male fertility in Arabidopsis

The role of JA in anther maturation was firstly established by showing that the triple fatty acid desaturase fad3-2 fad7-2 fad 8 mutant was male sterile and that fertility could be recovered by treatment with exogenous JA (McConn and Browse 1996). Similar effects were also observed for JA biosynthetic mutants: 12-oxophytodienoic acid reductase3 mutants (opr3, Stintzi and Browse 2000; dde1, Sanders et al. 2000), dadl lipase mutants (Ishiguro et al. 2001) and, finally, aos mutants (Park et al. 2002; dde2-2, von Malek et al. 2002). The role of LOX isoforms in male fertility has not been examined previously, and here we demonstrated that among all of the Arabidopsis 13-LOXs, LOX3 and LOX4 are the isoenzymes necessary for anther maturation, whereas LOX2 and LOX6 are not necessary for this process and do not contribute to male fertility. LOX3 and LOX4 share a nearly identical substrate binding pocket (Fig. 1b), and act redundantly in JA synthesis to ensure male fertility. In fact, single lox 3 and lox4 mutants are fertile and so are double mutants lox3 lox4 complemented either with LOX3 or LOX4 (Fig. 4). The next closest LOX from a sequence point of view is LOX6 (Bannenberg et al. 2009, and Fig. 1), but lox6 single mutants (Suppl. Fig. 3) as well as lox3 lox6 and lox4 lox6 double mutants are all fertile (Suppl. Fig. 4) indicating that LOX6 plays no major role in male fertility. LOX2 is phylogenetically the most diverse among the Arabidopsis 13-LOXs. Plants homozygous for a lox2 null allele are fertile and a major role of this LOX in the vegetative tissues of Arabidopsis was found instead to be in the synthesis of arabidopsides (Glauser et al. 2009; Seltmann et al. 2010). 


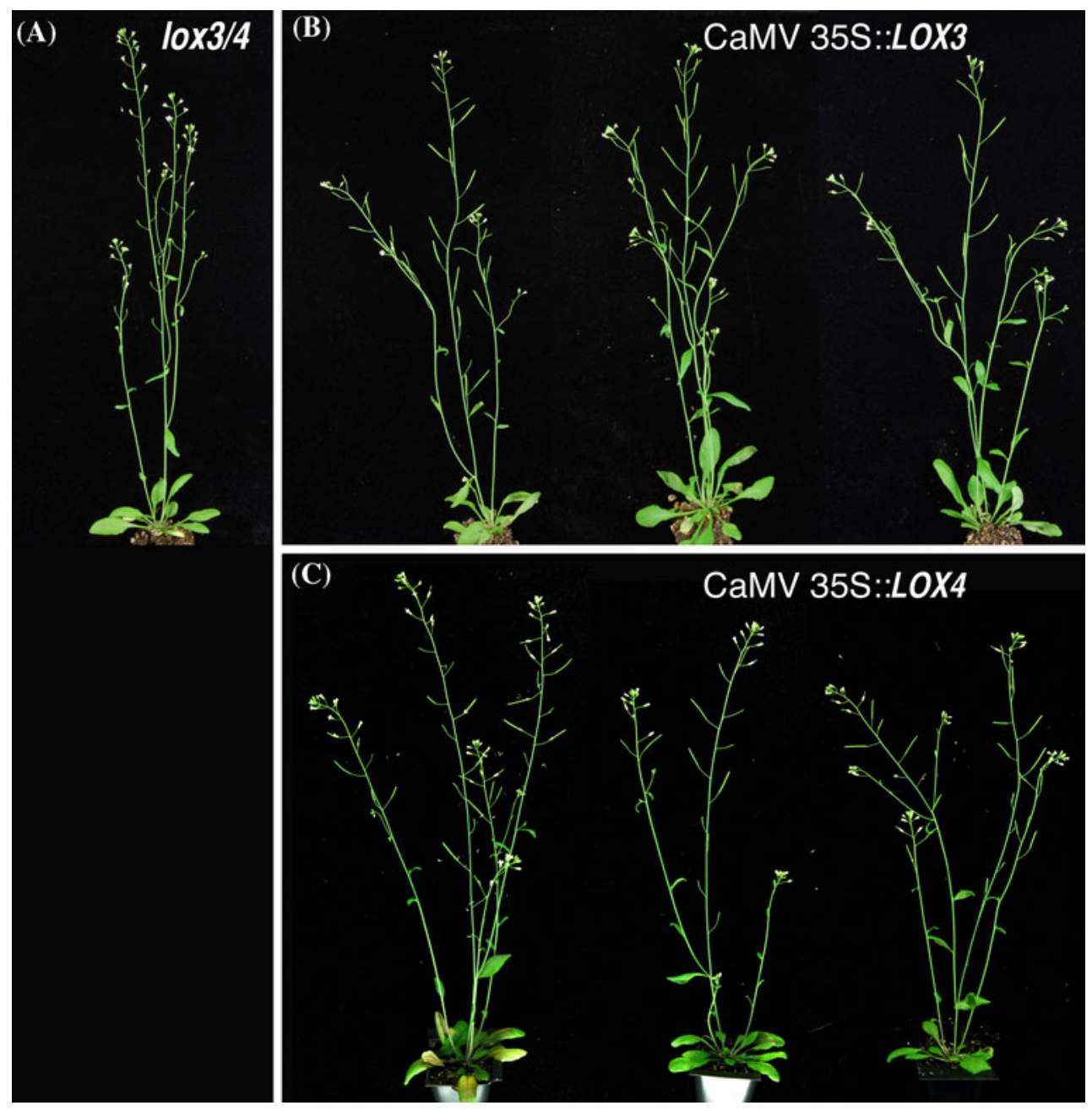

Fig. 4 Phenotype of the double mutant lox 3 lox 4 and complemented lines. a lox3 lox4; note the absence of siliques. b T1 generation of lox3 lox4 lines transformed with CaMV35S::AtLOX3. c T1 generation of lox3 lox4 lines transformed with CaMV35S::AtLOX4

The effects on male fertility that we observed in lox3 lox4 mutants were found to be the same as those reported for other Arabidopsis jasmonate synthesis and perception mutants (Feys et al. 1994; Ishiguro et al. 2001; Park et al. 2002; Sanders et al. 2000; Stintzi and Browse 2000; von Malek et al. 2002), that is an impairment in anther filament elongation, anther dehiscence, and pollen viability. Through further examination of lox3 lox4 double mutants as well as coil-1, aos, dadl and opr3 mutants, we found that these effects were systematically observable in all flowers except the terminal one (and rarely, in the penultimate flower). We also studied inflorescence growth in lox3 lox4 double mutants compared to aos, WT, and 13LOX single mutants. After flowering, lox3 lox4 and aos plants were taller than WT, and developed more floral shoots and more flowers. Instead 13-LOX single mutants developed in a similar way as the WT (Fig. 5). Thus, lox3 lox4 mutations affect many aspects of plant growth. lox3 lox4 double mutant inflorescences terminate in abnormal flowers

In WT plants, inflorescence meristematic activity invariably terminates with the production of unopened buds (Fig. 6a). This has been observed in the laboratory and in each of the 96 inflorescences of wild Arabidopsis thaliana collected in the field. In contrast, the terminal flower of lox3 lox4 double mutants develops abnormally (Fig. 6e-g), this flower does not develop petals, nor sepals, has a reduced number of stamens, and shows carpelloid and staminode structures. This same structure was then observed in a variety of other JA synthesis and perception mutants (dad1, aos, opr3 and coil-1). It seems that in all these male sterile mutants the signal for correctly terminating meristematic activity is missing, and the inflorescence meristem undergoes irreversible differentiation, terminating in an abnormal flower. We noticed that the 


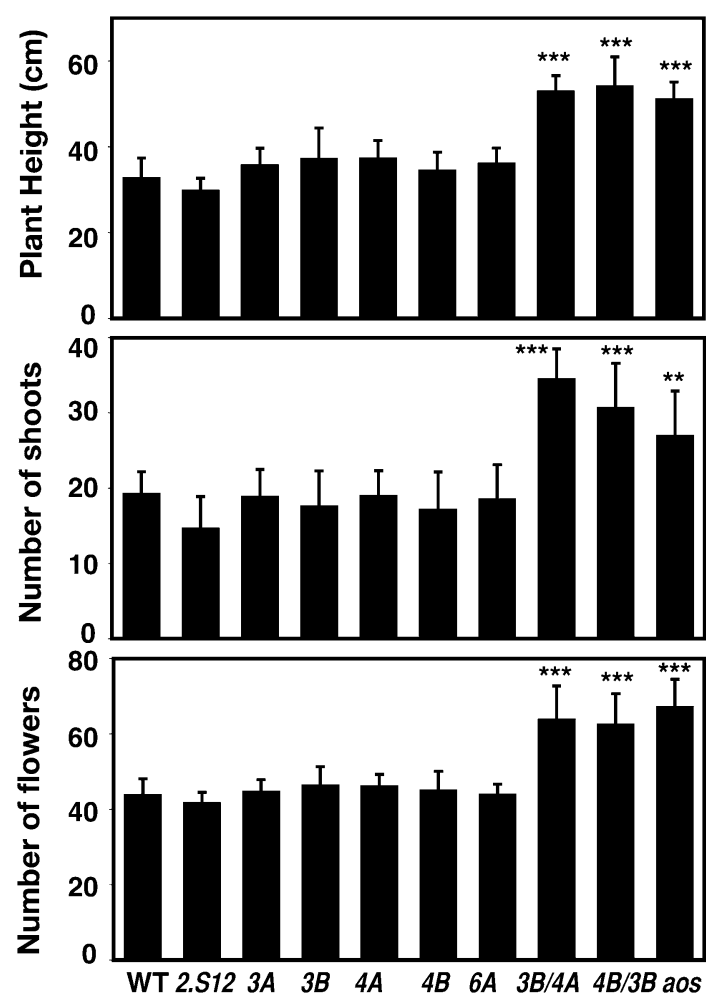

Fig. 5 Morphology of $L O X$ mutants, 9 weeks old. a Plants height. b Number of floral shoots. c Number of flowers. 2.S12 is the silenced LOX2 line. 3A, 3B, 4A, 4B, 6A, 3B/4A, 4B/3B correspond to the respective lines: $\operatorname{lox} 3 A$, lox $3 B$, lox $4 A$, lox $4 B$, lox $6 A$, double mutant lox $3 B$ lox $4 A$, lox $4 B$ lox $3 B$. Each bar represents an average of 12 measurements. Significance level of the $t$-test, $* *=P<0.01$; $* * *=P<0.001$

phenotype of this aberrant flower resembles that of the apetala2 mutant (ap2; Bowman et al. 1989; Jofuku et al. 1994). In ap2 mutants the phenotype is linked to the derepression of the homeotic gene AGAMOUS (AG; Drews et al. 1991; Bomblies et al. 1999). Similarly to the double mutant lox3 lox4, the inflorescence of $A G$ over-expressing plants also terminated with the production of fully developed abnormal flower consisting of a few carpelloid organs (Mizukami and Ma 1997).

Jasmonates indirectly control global proliferative arrest

Interestingly, Arabidopsis plants with a similar terminal flower phenotype to that of the lox 3 lox 4 double mutants have been observed in a different context. The male sterile1-1 (ms 1-1) mutant shows an aberrant terminal flower phenotype (Hensel et al. 1994). Moreover, a similar phenotype can be produced in WT plants through the systematic removal of developing fruits (Hensel et al. 1994, and Fig. $6 b-d)$. Developing fruits control the capacity of inflorescence meristems to produce additional flowers and, in Arabidopsis, a threshold of greater than $30 \%$ of WT seed

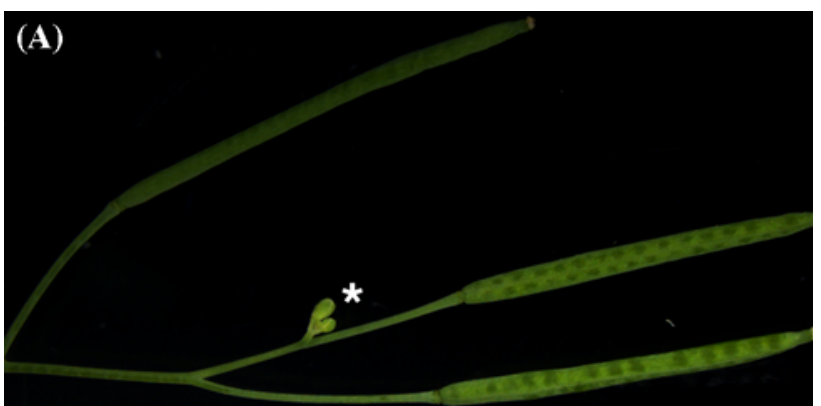

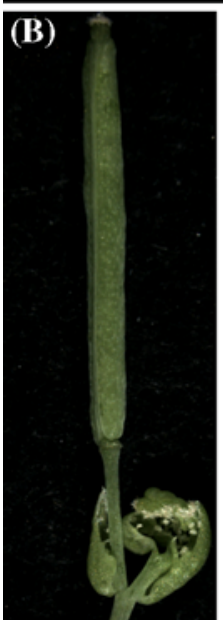
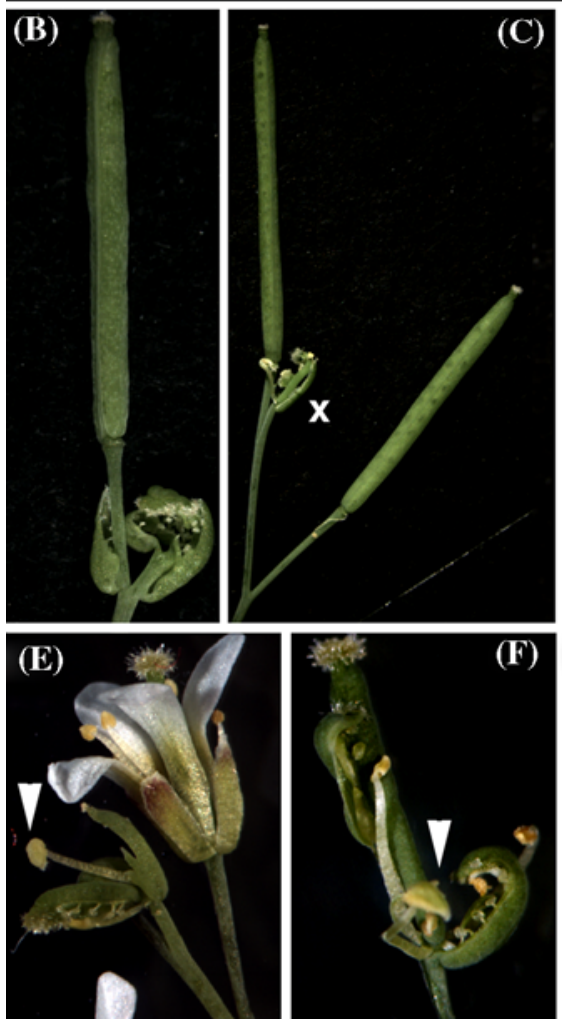

F)

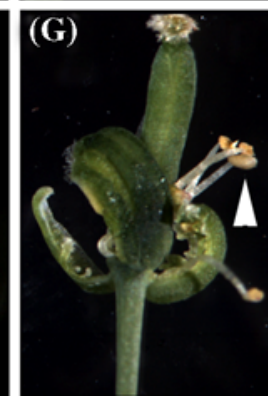

Fig. 6 Apical flower arrest. a WT (Col-O), the asterisk shows the terminal flower buds; b, c ultimate flower of WT (Col-O) plant in which fruits where surgically removed; $\mathbf{d}$ enlargement of the terminal flower is indicated by $X$ in (c); $\mathbf{e}, \mathbf{f}, \mathbf{g}$ three distinct ultimate flowers of lox3 lox4 double mutant. Arrowheads indicate staminode structures

per silique is necessary to induce an unknown signal that causes what is termed as 'global proliferative arrest' (GPA) in the inflorescence (Hensel et al. 1994). In order for GPA to operate correctly, a type of systemic signal must move over the comparatively long distances from the developing siliques to the apex. Mutants in the production or perception of other plant hormones, auxin, ethylene, abscissic acid and gibberellin were tested by Hensel et al. and found to have no effect on GPA (Hensel et al. 1994). Our findings show that, by assuring seed production, jasmonates indirectly determine the fate of the inflorescence meristem. In the absence of JA, no seed is set and the apex grows to a greater height than in the WT, terminating in a fully differentiated structure. In conclusion, this study shows that 
LOX3 and LOX4 control male fertility and inflorescence structure in Arabidopsis.

Acknowledgments We are grateful to Nicolas Guex for helping with sequence analysis and helpful comments, Shunping Yan for some photographs, Karolina Pajerowska-Mukthar and Ivan Acosta for constructive discussion. Funding was provided by NSF2010 grant to XD, Swiss NSF grant 3100A0_122441 to EEF, and by a Bourse pour l'Egalité des Chances at the University of Lausanne to DC. Author contributions: DC performed most experiments, GW prepared transgenic 35S::lox4 plants. DC, XD and EEF conceived experiments and DC and EEF wrote the paper, EEF made field observations.

\section{References}

Acosta IF, Laparra H, Romero SP, Schmelz E, Hamberg M, Mottinger JP, Moreno MA, Dellaporta SL (2009) tasselseed1 is a lipoxygenase affecting jasmonic acid signaling in sex determination of maize. Science 323:262-265

Andreou A, Feussner I (2009) Lipoxygenases: structure and reaction mechanism. Phytochemistry 70:1504-1510

Avanci NC, Luche DD, Goldman GH, Goldman MH (2010) Jasmonates are phytohormones with multiple functions, including plant defense and reproduction. Genet Mol Res 9:484-505

Bannenberg G, Martínez M, Hamberg M, Castresana C (2009) Diversity of the enzymatic activity in the lipoxygenase gene family of Arabidopsis thaliana. Lipids 44:85-95

Bell E, Creelman RA, Mullet JE (1995) A chloroplast lipoxygenase is required for wound-induced jasmonic acid accumulation in Arabidopsis. Proc Natl Acad Sci USA 92:8675-8679

Bomblies K, Dagenais N, Weigel D (1999) Redundant enhancers mediate transcriptional repression of AGAMOUS by APETALA2. Dev Biol 216:260-264

Boutet E, Lieberherr D, Tognolli M, Schneider M, Bairoch A (2007) UniProtKB/Swiss-Prot. Methods Mol Biol 406:89-112

Bowman JL, Smyth DR, Meyerowitz EM (1989) Genes directing flower development in Arabidopsis. Plant Cell 1:37-52

Brioudes F, Joly C, Szécsi J, Varaud E, Leroux J, Bellvert F, Bertrand C, Bendahmane M (2009) Jasmonate controls late development stages of petal growth in Arabidopsis thaliana. Plant $\mathrm{J}$ 60:1070-1080

Browse $\mathbf{J}$ (2009) The power of mutants for investigating jasmonate biosynthesis and signaling. Phytochemistry 70:1539-1546

Curtis MD, Grossniklaus U (2003) A gateway cloning vector set for high-throughput functional analysis of genes in planta. Plant Physiol 133:462-469

Drews GN, Bowman JL, Meyerowitz EM (1991) Negative regulation of the Arabidopsis homeotic gene AGAMOUS by the APETALA2 product. Cell 65:991-1002

Farmer EE, Dubugnon L (2009) Detritivorous crustaceans become herbivores on jasmonate-deficient plants. Proc Natl Acad Sci U S A $106: 935-940$

Feys B, Benedetti CE, Penfold CN, Turner JG (1994) Arabidopsis mutants selected for resistance to the phytotoxin Coronatine are male sterile, insensitive to methyl jasmonate, and resistant to a bacterial pathogen. Plant Cell 6:751-759

Fonseca S, Chico JM, Solano R (2009) The jasmonate pathway: the ligand, the receptor and the core signalling module. Curr Opin Plant Biol 12:539-547

Glauser G, Dubugnon L, Mousavi SAR, Rudaz S, Wolfender JL, Farmer EE (2009) Velocity estimates for signal propagation leading to systemic jasmonic acid accumulation in wounded Arabidospsis. J Biol Chem 284:34506-34513

Guex N, Peitsch MC (1997) SWISS-MODEL and the SwissPdbViewer: an environment for comparative protein modeling. Electrophoresis 18:2714-2723

Hensel LL, Nelson MA, Richmond TA, Bleecker AB (1994) The fate of inflorescence meristems is controlled by developing fruits in Arabidopsis. Plant Physiol 106:863-876

Ishiguro S, Kawai-Oda A, Ueda J, Nishida I, Okada K (2001) The defective in anther dehiscience gene encodes a novel phospholipase A1 catalyzing the initial step of jasmonic acid biosynthesis, which synchronizes pollen maturation, anther dehiscence, and flower opening in Arabidopsis. Plant Cell 13:2191-2209

Ito T, Ng KH, Lim TS, Yu H, Meyerowitz EM (2007) The homeotic protein AGAMOUS controls late stamen development by regulating a jasmonate biosynthetic gene in Arabidopsis. Plant Cell 19:3516-3529

Jofuku KD, den Boer BG, Van Montagu M, Okamuro JK (1994) Control of Arabidopsis flower and seed development by the homeotic gene APETALA2. Plant Cell 6:1211-1225

Li L, Li C, Howe GA (2001) Genetic analysis of wound signaling in tomato. Evidence for a dual role of jasmonic acid in defense and female fertility. Plant Physiol 127:1414-1417

Mandaokar A, Browse J (2009) MYB108 acts together with MYB24 to regulate jasmonate-mediated stamen maturation in Arabidopsis. Plant Physiol 149:851-862

McConn M, Browse J (1996) The critical requirement for linolenic acid is pollen development, not photosynthesis, in an Arabidopsis mutant. Plant Cell 8:403-416

Minor W, Steczko J, Stec B, Otwinowski Z, Bolin JT, Walter R, Axelrod B (1996) Crystal structure of soybean lipoxygenase L-1 at $1.4 \mathrm{~A}$ resolution. Biochemistry $35: 10687-10701$

Mizukami Y, Ma H (1997) Determination of Arabidopsis floral meristem identity by AGAMOUS. Plant Cell 9:393-408

Odell JT, Nagy F, Chua NH (1985) Identification of DNA sequences required for activity of the cauliflower mosaic virus $35 \mathrm{~S}$ promoter. Nature 313:810-812

Park JH, Halitschke R, Kim HB, Baldwin IT, Feldmann KA, Feyereisen R (2002) A knock-out mutation in allene oxide synthase results in male sterility and defective wound signal transduction in Arabidopsis due to a block in jasmonic acid biosynthesis. Plant J 31:1-12

Riemann M, Riemann M, Takano M (2008) Rice JASMONATE RESISTANT 1 is involved in phytochrome and jasmonate signalling. Plant Cell Environ 31:783-792

Sanders PM, Lee PY, Biesgen C, Boone JD, Beals TP, Weiler EW, Goldberg RB (2000) The Arabidopsis DELAYED DEHISCENCE1 gene encodes an enzyme in the jasmonic acid synthesis pathway. Plant Cell 12:1041-1061

Schneider C, Pratt DA, Porter NA, Brash AR (2007) Control of oxygenation in lipoxygenase and cyclooxygenase catalysis. Chem Biol 14:473-488

Seltmann MA, Stingl NE, Lautenschlaeger JK, Krischke M, Mueller MJ, Berger S (2010) Differential impact of lipoxygenase 2 and jasmonates on natural and stress-induced senescence in Arabidopsis thaliana. Plant Physiol 152:1940-1950

Skrzypczak-Jankun E, Bross RA, Carroll RT, Dunham WR, Funk MO Jr (2001) Three-dimensional structure of a purple lipoxygenase. J Am Chem Soc 123:10814-10820

Stintzi A, Browse J (2000) The Arabidopsis male-sterile mutant, opr3, lacks the 12-oxophytodienoic acid reductase required for jasmonate synthesis. Proc Natl Acad Sci U S A 97:10625-10630

von Malek B, van der Graaff E, Schneitz K, Keller B (2002) The Arabidopsis male-sterile mutant dde2-2 is defective in the ALLENE OXIDE SYNTHASE gene encoding one of the key 
enzymes of the jasmonic acid biosynthesis pathway. Planta 216:187-192

Wasternack C (2007) Jasmonates: an update on biosynthesis, signal transduction and action in plant stress response, growth and development. Ann Bot 100:681-697
Weigel D, Glazebrook J (2002) How to transform Arabidopsis. In: Arabidopsis: a laboratory manual. Cold Spring Harbor Laboratory Press, Cold Spring Harbor, NY 